\title{
Spinal versus Epidural Anesthesia for Vesico-vaginal Fistula Repair Surgery in a Rural Sub-Saharan African Setting
}

B. Inipavudu ${ }^{1}$; G. Mitterschiffthaler ${ }^{2}$; W. R. Hasibeder ${ }^{3}$; M. W. Dünser ${ }^{4}$

${ }^{1}$, Department of Anesthesia and Intensive Care Medicine, DOCS Hospital, Goma/Democratic Republic of the Congo; ${ }^{2}$, Department of Anesthesia and Intensive Care Medicine, Innsbruck Medical University, Anichstrasse 35, Innsbruck/Austria; ${ }^{3}$, Department of Anesthesia and Intensive Care Medicine, Krankenhaus der Barmherzigen Schwestern, Schlossberg 1, Ried im Innkreis/Austria; ${ }^{4}$, Department of Intensive Care Medicine, University Hospital Bern, Bern/Switzerland

Address for Correspondence:

Martin W. Duenser, M.D.; Department of Intensive Care Medicine, University Hospital Bern, Bern/Switzerland; Tel.: 0041-31-632-4144, Fax: 0041-31-632-9644, Email:

Martin.Duenser@uibk.ac.at

Running Title: $\quad$ Spinal vs. Epidural Anesthesia for VVF Surgery

Word count: $\quad 1541$ words (excl. abstract and references)

No author has a conflict of interest in regards of any drug or technique discussed in this manuscript. 


\section{ABSTRACT}

Study Objective: To compare spinal anesthesia with epidural anesthesia in patients undergoing surgery for vesico-vaginal fistula (VVF) repair.

Design: Non-randomized, prospective clinical trial.

Setting: Rural African Hospital with 165 beds.

Patients: Sixty patients undergoing VVF repair surgery with ASA classification I or II.

Interventions: Spinal (1.5-2 mL hyperbaric bupivacaine 0.75\%) or lumbal epidural (20-24 $\mathrm{mL}$ bupivacaine $0.5 \%$ ) anesthesia.

Measurements: Demographics, quality of anesthesia, duration of postoperative analgesia, as well as pre-, intra-, and postoperative data. Data were compared between study groups using unpaired student's $t$-test for continuous variables, and Chi-square and Fisher's exact tests for categorical data.

Main Results: Thirty patients were included in each study group. Quality of anesthesia was different between groups ( $p=0.009$ ). Good anesthesia quality was significantly more frequent in the spinal (86.7\%) than the epidural group (50\%) $(p=0.005)$. The quality of postoperative analgesia was comparable $(p=0.347)$. There were no differences in haemodynamic parameters before, during, and after surgery between groups.

Conclusions: Spinal anesthesia proved to be the superior anaesthetic technique for VVF repair surgery when compared with epidural anesthesia. In the rural, Sub-Saharan African setting, spinal anesthesia seems to have more advantages than epidural anesthesia for this type of surgery.

Word count: 194 words 


\section{INTRODUCTION}

Vesico-vaginal fistulas (VVF) are a widely unrecognized, but frequent problem of women's health care, particularly in developing countries $(1,2)$. While in Western countries, the majority of VVFs occur after complicated pelvic surgery, over $90 \%$ of VVF in the developing world are of obstetric origin and result from prolonged obstructed labour (3). In areas of civil war, such as the North Kivu province in the Democratic Republic of the Congo during the last ten years, sexual trauma due to forced intercourse is another important cause of VVF (4). Surgical management of VVF repair mostly includes transvaginal therapies, which have recently been standardized (5). In contrast, almost no data on the optimal anesthetic technique for VVF surgeries exist.

Because of its simplicity and decreased need for oxygen, spinal and epidural anesthesia are frequently performed for gynecelogic and obstetric surgery in Sub-Saharan Africa (6). While hypotension, headache, and vomiting may complicate spinal anesthesia, the risks of epidural anesthesia are, among others, inadvertent intrathecal or intravascular injection of local anesthetics (7).

This prospective clinical study compares spinal and epidural anesthesia for VVF surgery in a rural Sub-Saharan African setting. Our hypothesis was that there would be no difference between the two anesthesia techniques. 


\section{MATERIALS AND METHODS}

The trial was conducted at the DOCS hospital in Goma, North Kivu Province in the Democratic Republic of the Congo. The study protocol was approved by the local scientific committee which coordinates scientific efforts and medical education, and also serves as an ethical committee.

\section{Setting}

The DOCS hospital in Goma is a 165 bed hospital specialized on orthopedic, gynecological, and general surgery. In the year 2005, a total of 813 surgical interventions were performed. With 211 cases, VVF repair surgeries made up one quarter of all operations. Owing to financial support by the international DOCS association, the hospital is equipped well when compared to other hospitals in the region (6). Anesthetic equipment in each of the four operation theatres consists of an anesthesia machine capable to deliver inhalation anesthesia and a monitor able to measure ECG, non-invasive blood pressure, oxygen saturation, temperature, and endtidal carbon dioxide.

\section{Patients}

All patients undergoing VVF repair surgery during the time from January until December 2005 were eligible for study entry. Because various anesthetic techniques are used by different anesthetists in the hospital, only patients anaesthetized by I.B. were included into the study protocol. Exclusion criteria were age below 16 years, Amercian Society of Anesthesiologists Classification of III or higher, unwillingness of the patient to undergo regional anesthesia, and contraindications to spinal or epidural anesthesia. 


\section{Study Groups}

After study inclusion, patients were allocated to a spinal or an epidural anesthesia group. Because of the intermittent availability of either bupivacaine $0.75 \%$ (used for spinal anesthesia) or bupivacaine $0.5 \%$ (used for epidural anesthesia) the study group allocation could not be randomized according to a computerized scheme, but was given by the availability of the anesthetic drugs.

For spinal anesthesia, 1.5 to $2 \mathrm{~mL}$ (11.25-15 mg) of hyperbaric bupivacaine $0.75 \%$ were administered. Using G25/G22 Quincke or Whitetacre needles, puncture was performed at the level of L3/L4 or L4/L5 resulting in a cutaneous sensory loss up to T12/L1. For epidural anesthesia, 20-24 mL (100-120 mg) of isobaric bupivacaine $0.5 \%$ were administered. Using G17/18 Tuohy needles, the same spinal level was used resulting in a comparable sensory level at T12/L1.

\section{Data Documentation}

In all study patients, age, body mass index, pre-existent diseases, time of onset of anesthesia, quality of anesthesia, duration of surgery, duration and quality of postoperative analgesia, occurrence of adverse events during the intra- or postoperative period, as well as length of hospitalization, and patient outcome at hospital discharge were documented.

Hemodynamic parameters recorded were highest heart rate and lowest mean arterial blood pressure before, during, and after surgery; additionally, type and amount of intraoperative fluids administered. The quality of anesthesia was categorized as good (no need for additional sedative or analgesics), moderate (need for additional sedative or analgesics), and poor (need for general anesthesia). Similarly, the quality of postoperative analgesia was categorized as good (no need for additional analgesics), moderate (need for additional analgesics), and poor (immediate need for analgesics). Duration of postoperative analgesia 
was defined as the time from the end of surgery until the first analgesic had to be administered.

\section{Statistical Analysis}

Since this was a pilot study, no power analysis could be performed and a sample size of 30 patients in each group was prospectively agreed. The primary study endpoint was to evaluate differences in the quality of anesthesia and postoperative analgesia between spinal and epidural anesthesia. The secondary study endpoint was to evaluate differences in intraand postoperative hemodynamic parameters as well as the incidence of adverse events between the two anesthetic techniques.

Shapiro Wilk’s and Kolmogorov Smirnov tests were used to check for normality distribution of data. Data were compared between study groups using unpaired student's $t$-test for continuous variables, and Chi-square and Fisher's exact tests for categorical data. $P$-values $<0.05$ were considered to indicate statistical significance. All data are presented as mean values $\pm \mathrm{SD}$, if not indicated otherwise. 


\section{RESULTS}

Table 1 presents characteristics of all study patients. There were no differences between study groups.

The onset of anesthesia occurred significantly faster in patients with spinal (12.9 \pm 3.5 min) than with epidural anesthesia $(21.8 \pm 4.2 \mathrm{~min})(p<0.001)$. The quality of anesthesia was significantly different between study groups ( $p=0.009$ ) (Figure 1). Good anesthesia quality could be achieved in significantly more patients in the spinal (26/30, 86.7\%) than in the epidural group (15/30, 50\%) ( $p=0.005)$. There was a non-significant trend towards a higher frequency of moderate anesthesia quality in the epidural group (10/30, 33.3\%) vs. the spinal group (3/30, 10\%) ( $p=0.057)$. While five patients in the epidural group (16.7\%) required general anesthesia, one patient had to undergo general anesthesia in the spinal group (3.3\%) ( $p=0.195)$. The quality of postoperative analgesia was not different between groups $(p=0.347)$. Time to first need of postoperative analgesics was comparable between groups (spinal anesthesia, 167 \pm 48 min; epidural anesthesia, 181 \pm 64 min; $p=0.353$ ).

There were no differences in hemodynamic parameters before, during, and after surgery between the spinal and epidural anesthesia group (Table 2). In both groups, a significant decrease in mean arterial blood pressure occurred during anesthesia $(p<0.001$ each).

During the intra- and postoperative period, nine adverse events occurred in the spinal anesthesia group (nausea, $n=2$; vomiting, $n=4$; agitation, $n=1$; shivering, $n=1$; backache, $n=1$ )., while six occurred in the epidural anesthesia group (nausea, $n=1$; vomiting, $n=3$; agitation, $n=1$; shivering, $n=1)$. This difference was not significant $(p=0.908)$. 


\section{DISCUSSION}

In this rural, Sub-Saharan African setting, spinal anesthesia proved to result in significantly better anesthesia quality for VVF repair surgery when compared with epidural anesthesia. There were no differences in hemodynamic parameters or the incidence of adverse events between groups.

Particularly in developing countries, further advantages of spinal anesthesia seem to exist: First, the significantly higher rate of good anesthesia quality resulted in the less frequent need for additional drugs which are rare in most Subs-Saharan African hospitals. Since the majority of hospitals in developing countries are not as well equipped in terms of intraoperative monitoring and oxygen availability as the study centre (6), the use of spinal anesthesia may further improve perioperative patient safety. Second, the significantly faster onset of anesthesia in the spinal when compared to the epidural anesthesia group allows for a higher number of surgeries to be done in a given time. This is of particular interest in centers specialized on VVF repair surgery such as the DOCS hospital in Goma, where hundreds of women wait to be operated while others continue to arrive from distant villages. Third, while 1.5-2 mL of local anaesthetic were used for spinal, 20-24 mL were needed for epidural anesthesia in this study. The lower amount of local anesthetic required may thus help to decrease anesthesia costs for VVF repairs surgery in developing countries. Fourth, the spinal anesthesia technique was easy to be learned and performed $(8,9)$.

So far, two studies on anesthesia techniques for VVF repair surgery have been published $(4,10)$. Based on his experience in 38 women, Thomson recommended the combination of spinal and epidural anesthesia to be the anesthetic technique of choice for VVF repair surgery (4). Spinal anesthesia with pethidine $(1 \mathrm{mg} / 5 \mathrm{~kg})$ produced good anesthesia quality in thirty patients undergoing VVF repair surgery (10). Even though the current literature does not allow one to conclude whether spinal anesthesia is superior to epidural anesthesia $(11,12)$, our results support spinal anesthesia $(13,14)$. 
When interpreting the results of this study important limitations need to be considered. Since the study was not randomized and patients were only included if cared for by a selected anesthetist, the authors cannot exclude a certain selection bias. However, since there were no differences in demographic and patient data, this seems to be unlikely. The sample size of our study was too small to reliably assess differences in the incidence of adverse events between spinal and epidural anesthesia. Although no differences in the occurrence of adverse events were observed, we may have not been able to adequately reflect the benefit:risk ratio of the two anesthetic techniques.

In this pilot study, spinal anesthesia proved to be the better anesthetic technique for VVF repair surgery when compared with epidural anesthesia in a rural, Sub-Saharan African setting. 


\section{REFERENCES}

1. Hilton P. Vesico-vaginal fistulas in developing countries. Int J Gynecol Obstet 2003;82:285-295.

2. Wall LL. Obstetric fistulas in Africa and the developing world: New efforts to solve an age-old problem. Women's Health Issues 1996;6:229-234.

3. Steiner AK. The problem of post-partum fistulas in developing countries. Acta Trop 1996;62:217-223.

4. Thomson KD. A use for combined spinal epidural anesthesia at last! Anesthesia 2002;57:618.

5. Angioli R, Penalver M, Muzii L, et al. Guidelines of how to manage vesicovaginal fistola. Crit Rev Oncol Hematol 2003;48:295-304.

6. Dünser M, Baelani I, Ganbold L. The speciality of anesthesia outside Western medicine. With special consideration of personal experiences in the Democratic Republic of the Congo and Mongolia. Anaesthesist 2006;55:118-132.

7. Arndt M, Benad G. The risks of anesthesia in obstetric interventions. Anaesthesiol Reanim 1994;19:88-94.

8. Konrad C, Schüpfer G, Wietlisbach M, Gerber H. Learning manual skills in anesthesiology: Is there a recommended number of cases for anesthetic procedures? Anesth Analg 1998;86:635-639.

9. Kestin I. A statistical approach to measuring the competence of anaesthetic trainees at practical procedures. Brit J Anaesthesia 1995;75:805-809.

10. Saissy JM. Experience with spinal anesthesia using pethidine in the surgery of vesicovaginal fistola in African women (30 cases). Med Trop (Mars) 1984;44:61-64.

11. Ng K, Parsons J, Cyna AM, Middelton P. Spinal versus epidural anaesthesia for caesarean section. Cochrane Database Syst Rev 2004;(2):CD003765. 
12. Veneziani A, De Tommaso O. Spinal or epidural anaesthesia for caesarean section? Compared opinions. Minerva Anesthesiol 2001;67:169-174.

13. Albani A, Renghi A, Ciarlo M, Avallone V, Toscano M. Peridural anesthesia versus subarachnoid anesthesia in cesarean section. Prospective clinical study. Minerva Anestesiol 1998;64:387-391.

14. Bhattarai B, Rahman TR, Sah BP, Singh SN. Central neural blocks: a quality assessment of anaesthesia in gynaecological surgeries. Nepal Med Coll J 2005;7:93-96. 
Table 1. Characteristics of Study Patients.

\begin{tabular}{|c|c|c|c|}
\hline & Spinal Anaesthesia & Epidural Anaesthesia & $p$-value \\
\hline$n$ & 30 & 30 & \\
\hline Age, years & $32 \pm 13$ & $30 \pm 13$ & 0.515 \\
\hline BMI, kg.m ${ }^{-2}$ & $22 \pm 2$ & $23 \pm 2$ & 0.245 \\
\hline Pre-existent Diseases, n/\% & & & 0.667 \\
\hline none & $19 / 63.43 \%$ & $18 / 60 \%$ & \\
\hline Chronic Tuberculosis & $0 / 0 \%$ & $1 / 3.3 \%$ & \\
\hline Past Cesarean Section or other Surgery & $5 / 16.7 \%$ & $8 / 26.7 \%$ & \\
\hline Other & $3 / 10 \%$ & $3 / 10 \%$ & \\
\hline Duration of Surgery, min & $92 \pm 41$ & $105 \pm 51$ & 0.311 \\
\hline Length of Hospital Stay, days & $25 \pm 5$ & $26 \pm 9$ & 0.477 \\
\hline Mortality at Hospital Discharge, n/\% & $0 / 0 \%$ & $1 / 3.3 \%$ & 1 \\
\hline
\end{tabular}

BMI, body mass index.

Parameters are given as mean values \pm SD, if not indicated otherwise. 
Table 2. Hemodynamic Parameters in Study Patients.

\begin{tabular}{|c|c|c|c|c|c|}
\hline & Spinal & Anesthesia & Epidural & I Anesthesia & $p$-value \\
\hline$n$ & & 30 & & 30 & \\
\hline \multicolumn{6}{|l|}{ Heart Rate, beats/min } \\
\hline Before & 83 & \pm 11 & 77 & \pm 12 & 0.059 \\
\hline During & 87 & \pm 15 & 83 & \pm 18 & 0.347 \\
\hline After & 86 & \pm 13 & 86 & \pm 14 & 0.433 \\
\hline \multicolumn{6}{|c|}{ Mean Arterial Pressure, mmHg } \\
\hline Before & 89 & \pm 8 & 91 & \pm 10 & 0.479 \\
\hline During & 76 & \pm 7 & 79 & \pm 11 & 0.150 \\
\hline After & 79 & \pm 9 & 80 & \pm 10 & 0.529 \\
\hline \multicolumn{6}{|c|}{ Intraoperative Fluid Amount, mL } \\
\hline Crystalloids & 1008 & \pm 247 & 980 & \pm 426 & 0.766 \\
\hline Colloid (Gelatine) $(n=5 / 6)$ & 636 & \pm 166 & 508 & \pm 163 & 0.230 \\
\hline
\end{tabular}

Parameters are given as mean values $\pm \mathrm{SD}$, if not indicated otherwise. 
Figure 1. Differences in Quality of Anesthesia and Quality of Postoperative Analgesia between Study Groups.

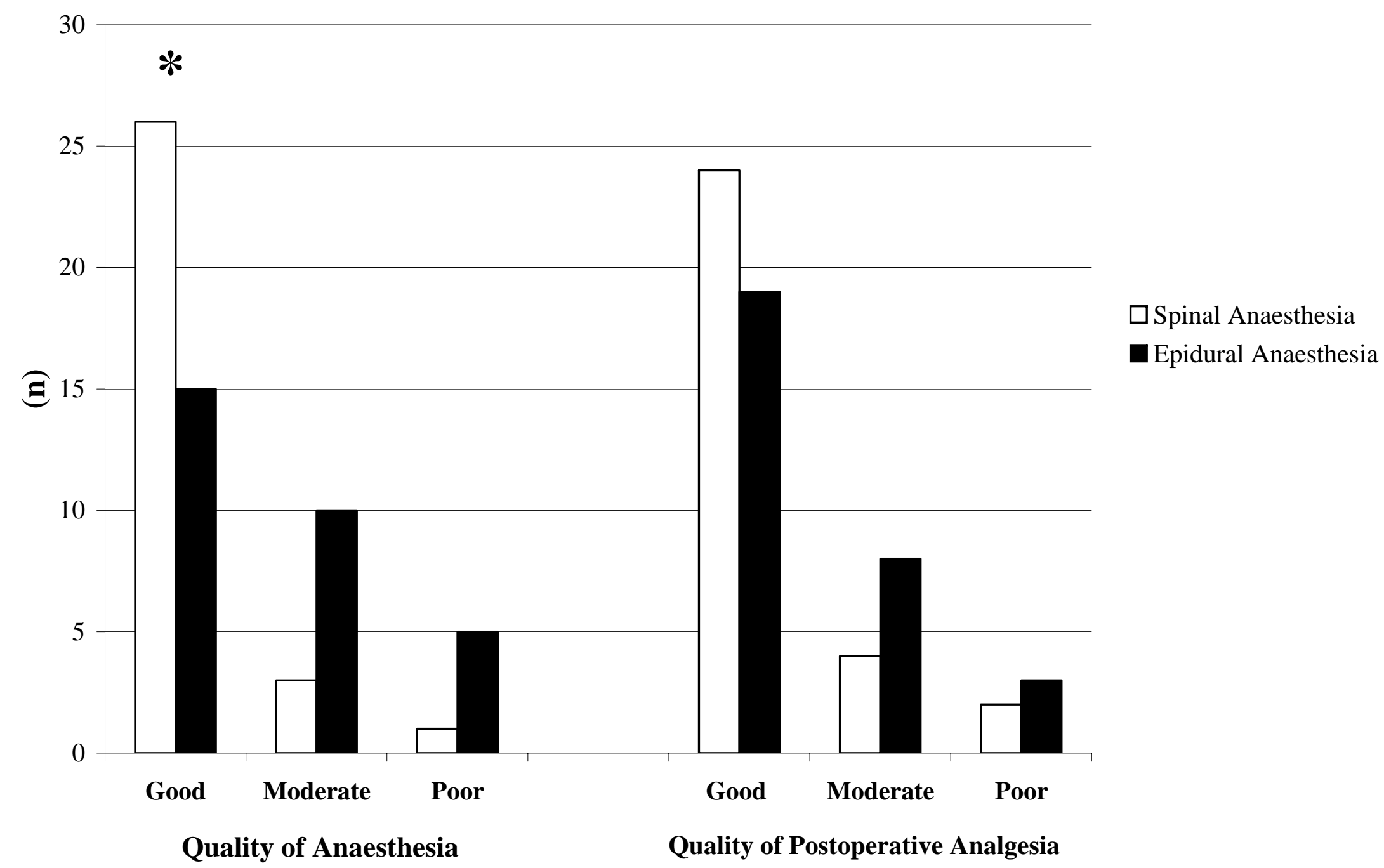

*, significant difference between spinal and epidural anesthesia. 